\title{
What does left atrial structural remodeling mean in cardiac resynchronization therapy?
}

\author{
Sung-Hee Shin
}

Division of Cardiology, Department of Internal Medicine, Inha University Hospital, Incheon, Korea

Received: August 8, 2020 Accepted: August 12, 2020

\section{Correspondence to} Sung-Hee Shin, M.D. Division of Cardiology, Department of Internal Medicine, Inha University Hospital, 27 Inhang-ro, Jung-gu, Incheon 22332, Korea Tel: $+82-32-890-2200$ Fax: $+82-32-890-2447$ E-mail: sshin@inha.ac.kr https://orcid.org/0000-00028306-9622

\section{See Article on Page 1125-1135}

Cardiac resynchronization therapy (CRT) improves left ventricular (LV) systolic function and clinical outcomes in select patients with heart failure, reduced LV ejection fraction and electrocardiographic evidence of electrical dyssynchrony. However, approximately one-third of patients do not respond to CRT, and researchers have investigated better predictive algorithms that can identify the most suitable patients before CRT implantation. While the Predictors of Response to CRT (PROSPECT) trial failed to show any benefit of echocardiographic and tissue Doppler-based evaluation of mechanical synchrony in selecting patients for CRT [1], many researchers are still trying to refine CRT selection more precisely, beyond the current guidelines. The beneficial effects of CRT can be attributed to LV reverse remodeling, a decrease in mitral regurgitation, and an improvement in LV diastolic dysfunction [2,3].

In this issue of the Korean Journal of Internal Medicine, Cho et al. [4] showed that a reduction in left atrial (LA) volume less than 1 year after CRT was associated with late LV reverse remodeling. Interestingly, the LA volume decreased only up to 1 year after CRT, whereas the LV volume decreased continuously during the follow-up period, which was a median of 30 months. In this study, the decrease in LA volume was related to a reduction in early mitral inflow velocity, among several echocardiographic parameters. This corresponds with previous studies reporting that CRT caused a reduction in LA volume, which was related to LV reverse remodeling and independently associated with improved clinical outcomes after device implantation [5-7]. Effective CRT could result in reduced LV filling pressure by decreasing inter- and intraventricular dyssynchrony. This can lead to a decrease in the early mitral inflow velocity and thus a decrease in LA volume resulting from lengthy exposure to abnormal LV filling pressure. Eventually, these changes would contribute to LV reverse remodeling. However, the causal relationship cannot be clearly elucidated from this study because an early favorable LV change itself can affect LA reverse remodeling, given the nature of the interaction between the LA and LV. A decrease in mitral regurgitation can also reduce LA size. These favorable effects of CRT on cardiac structure and function would translate into better clinical outcomes. In addition, a significant reduction in the risk of subsequent atrial tachyarrhythmia with LA 
structural remodeling can also lead to better outcomes [8]. However, it might be that reverse LA remodeling becomes ineffective after severe LA enlargement, given that previous data suggest that marked LA dilatation is associated with worse clinical and echocardiographic outcomes [9]. Initiation of CRT at the appropriate time might improve prognosis in patients with heart failure by decreasing chronic LA pressure overload earlier.

While guidelines have proposed that LA volume is a marker for evaluating LV diastolic function and estimating LV filling pressure, recent studies have shown the utility of LA functional parameters assessed by volumetric methods or speckle-tracking echocardiography as sensitive indicators of LV diastolic dysfunction and good predictors in patients with heart failure $[10,11]$. Despite not assessing LA functional parameters in the current study, other studies have demonstrated improved LA function after CRT and the possible utility of LA functional analysis in predicting CRT response $[12,13]$. Although studies of LA-LV interactions are needed to elucidate whether LA deformational indices provide measures of intrinsic LA function beyond LA structural remodeling and LV function, measuring LA strain using speckle-tracking echocardiography looks promising for assessing LA mechanics. Further studies are required to determine whether integrating LA structural or functional analysis provides additional valuable information in assessing CRT candidates and predicting CRT response in a broad clinical setting.

\section{Conflict of interest}

No potential conflict of interest relevant to this article was reported.

\section{REFERENCES}

1. Chung ES, Leon AR, Tavazzi L, et al. Results of the Predictors of Response to CRT (PROSPECT) trial. Circulation 2008;117:2608-2616.

2. Solomon SD, Foster E, Bourgoun M, et al. Effect of cardiac resynchronization therapy on reverse remodeling and relation to outcome: multicenter automatic defibrillator implantation trial: cardiac resynchronization therapy.
Circulation 2010;122:985-992.

3. Waggoner AD, Faddis MN, Gleva MJ, et al. Cardiac resynchronization therapy acutely improves diastolic function. J Am Soc Echocardiogr 2005;18:216-220.

4. Cho IJ, Uhm JS, Oh J, et al. Left ventricular response after cardiac resynchronization therapy is related to early left atrial volume reduction. Korean J Intern Med. 2020;35:1125-1135.

5. Kuperstein R, Goldenberg I, Moss AJ, et al. Left atrial volume and the benefit of cardiac resynchronization therapy in the MADIT-CRT trial. Circ Heart Fail 2014;7:154-160.

6. Verbrugge FH, Dupont M, Vercammen J, et al. Time from emerging heart failure symptoms to cardiac resynchronisation therapy: impact on clinical response. Heart 2013;99:314-319.

7. Yu CM, Fang F, Zhang Q, et al. Improvement of atrial function and atrial reverse remodeling after cardiac resynchronization therapy for heart failure. J Am Coll Cardiol 2007;50:778-785.

8. Brenyo A, Link MS, Barsheshet A, et al. Cardiac resynchronization therapy reduces left atrial volume and the risk of atrial tachyarrhythmias in MADIT-CRT (Multicenter Automatic Defibrillator Implantation Trial with Cardiac Resynchronization Therapy). J Am Coll Cardiol 2011;58:1682-1689.

9. Rossi L, Malagoli A, Piepoli M, et al. Indexed maximal left atrial volume predicts response to cardiac resynchronization therapy. Int J Cardiol 2013;168:3629-3633.

10. Thomas L, Marwick TH, Popescu BA, Donal E, Badano LP. Left atrial structure and function, and left ventricular diastolic dysfunction: JACC state-of-the-art review. J Am Coll Cardiol 2019;73:1961-1977.

11. Malagoli A, Rossi L, Bursi F, et al. Left atrial function predicts cardiovascular events in patients with chronic heart failure with reduced ejection fraction. J Am Soc Echocardiogr 2019;32:248-256.

12. Donal E, Tan K, Leclercq C, et al. Left atrial reverse remodeling and cardiac resynchronization therapy for chronic heart failure patients in sinus rhythm. J Am Soc Echocardiogr 2009;22:1152-1158.

13. Feneon D, Behaghel A, Bernard A, et al. Left atrial function, a new predictor of response to cardiac resynchronization therapy? Heart Rhythm 2015;12:1800-1806. 